Revista LIDER, 37(22), 2020, pp. 77-99

eISSN: 0719-526

ISSN: 0717-0165

\title{
Integración territorial como marginación: obstáculos para las zonas aisladas en Aysén y Magallanes, Chile
}

\author{
Territorial integration as marginalization: obstacles \\ for remote areas in Aysén and Magallanes, Chile
}

\author{
Álvaro Román \\ Universidad de Los Lagos, Chile.alvaro.roman@ulagos.cl
}

\section{RESUMEN}

Las zonas aisladas son territorios definidos por sus carencias y son analizados desde su distancia, sus problemas de conectividad o su falta de dinamismo económico. En Chile, este fenómeno es abordado mediante medidas de integración, principalmente a través de la institucionalidad pública y el mercado. Sin embargo, se presta menos atención a las definiciones realizadas desde los territorios para caracterizar las evidentes diferencias con los polos de desarrollo. Este artículo cuestiona la noción de integración territorial de estas zonas como un medio para promover su desarrollo y mejorar su calidad de vida. En particular, se exponen las omisiones y obstáculos que enfrentan territorios aislados al intentar cumplir objetivos definidos a la distancia. Basado en entrevistas semiestructuradas aplicadas en las comunas de Aysén y Cabo de Hornos, en el sur de Chile, se identifican los problemas cotidianos de habitar territorios extremos, con clima, conectividad y base económica desafiantes. También se releva las particularidades locales de estas comunas y sus posibilidades de actuar en pos de la integración de estas zonas en sus propios términos.

Palabras clave: Cabo de Hornos; compresión espacio-temporal; injusticia hermenéutica; integración territorial; lejanía

\section{ABSTRACT}

Remote areas are territories defined by their shortcomings, and are analyzed from their distance, their connectivity problems or their lack of economic dynamism. In Chile, this phenomenon is addressed through integration measures, mainly through public institutions and the market. However, less attention is paid to the definitions made from the territories to characterize the evident differences with poles of development. This article questions the notion of territorial integration of these areas as a means to promote their development and improve their quality of life. In particular, it exposes the omissions and obstacles faced by remote areas when trying to meet goals defined at a distance. Based on semi-structured interviews applied in the communes of Aysén and Cabo de Hornos, in Southern Chile, we identify daily problems of inhabiting extreme territories, including challenging climate, connectivity and economic base. The analysis also reveals local particularities of these communes and their possibilities of acting towards the integration of these areas on their own terms.

Key words: Cabo de Hornos; time-space compression; hermeneutic injustice; territorial integration; remoteness

Recibido: 12 de octubre de 2020. Aceptado: 16 de noviembre de 2020 . 


\section{Introducción}

La integración del territorio ha sido tomada como un medio para abordar los problemas impuestos por el aislamiento geográfico. Este último ha sido caracterizado principalmente desde sus aspectos negativos, en especial cuando impacta sobre definiciones de desarrollo construidas a partir del desempeño de diversos territorios bajo una misma escala, como lo es el nivel nacional (Arenas et al., 1999). Se trata de zonas en las que el dinamismo económico, la calidad de vida y el acceso a bienes y servicios son menores a los de áreas centrales, con grandes concentraciones de población y donde se sitúa la toma de decisiones políticas, administrativas y económicas (Garay, 2004; Soza-Amigo \& Correa, 2014) . Al ser abordadas a través de una mirada desde el centro se apunta a estrategias que aminoren las brechas con respecto a territorios con mejores desempeños, antes que a identificar sus particularidades y a definir distinciones que las releven como parte de su propio desarrollo. Así conceptualizado, el aislamiento se resuelve a través de la incorporación de estos territorios.

En Chile, la integración de las zonas aisladas al desarrollo nacional se ha centrado en estrategias que propicien la inversión privada a través de mecanismos tributarios y al mejoramiento de la conectividad a través de subsidios de transporte y de la construcción de vías terrestres. Dicho enfoque ha sido cuestionado por su rigidez. Por una parte, aplica un estándar a alcanzar para territorios muy diferentes entre sí, y todos ellos con diferencias notables respecto a las áreas centrales. Así, los objetivos planteados de manera estandarizada pueden ser inalcanzables para zonas que cuentan con menos recursos económicos, con instituciones locales debilitadas o con carencias técnicas (Amigo, 2017; Brigand et al., 2011; Núñez, Arenas, \& Salazar, 2011; Soza-Amigo \& Aroca, 2010). Además, dichos objetivos pueden incluso resultar irrelevantes para el contexto local. Por otra, no reconoce los avances ni las expectativas locales orientados a un desempeño diferenciado, que se centre en el bienestar local antes que en las ventajas que estos territorios entrañan para el resto del país (Soza-Amigo \& Aroca, 2010; Soza-Amigo \& Correa, 2014). Por ejemplo, en zonas de interés turístico puede haber incompatibilidades entre las apuestas por una dinámica económica estacional que requiere de la preservación del paisaje y el establecimiento de iniciativas productivas basadas en recursos naturales (Pons \& Rullan, 2020).

Del total de localidades en Chile, alrededor del $13,5 \%$ son consideradas aisladas debido a que, por sus características demográficas, de infraestructura y de acceso a servicios enfrentan dificultades para integrarse al ritmo de desarrollo del resto del país (Subsecretaría de Desarrollo Regional y Administrativo, 2012). En ellas se concentra el 1,1\% de la población nacional. Si bien el valor es bajo, contiene disparidades territoriales notables, pues mientras el o,1\% de la población de la región 
Metropolitana está aislada, en Aysén lo está el 11,4\%. Además, entre el 54\% y el 81\% de las localidades de las zonas extremas son aisladas. Son áreas diversas, que reúnen zonas de montaña, archipiélagos, islas oceánicas y territorios fronterizos. No constituyen una unidad territorial y sus carencias respecto a las zonas centrales son diferentes en cada caso. De manera paradójica, son áreas cuya comprensión más detallada suele quedar excluida, precisamente por lo difícil de acceder a ellas o de interpretar sus dinámicas (Ministerio de Desarrollo Social, 2018).

Lo anterior muestra que la integración de las zonas aisladas al territorio nacional se basa en supuestos que no están suficientemente demostrados. Evidencia de ello son los trabajos de Núñez y otros (2017) y Tapia (2015), quienes muestran las implicancias que la administración homogénea del territorio tiene sobre la vida cotidiana de quienes habitan territorios fronterizos. Residentes ligados a actividades tradicionales se ven involucrados en complejas relaciones caracterizadas por consideraciones geopolíticas y económicas, donde el bienestar local es invisibilizado. Entre los estudios insulares ocurre algo similar, con habitantes confinados en territorios rodeados por el mar, donde el día a día está marcado por la dependencia de recursos marinos y por la carencia de bienes y servicios, además de las dificultades de desplazamiento (Saavedra, 2016).

En Chile la integración territorial pasa por mecanismos definidos en las leyes 19.606 y 19.853, que establecen exenciones tributarias y subsidios a la contratación de mano de obra, respectivamente. Sin embargo, estas medidas no solo han mostrado ser insuficientes, sino también poco adecuadas porque se limitan a promover la incorporación de las zonas más remotas de Chile al tren de desarrollo definido desde la zona central del país, sin considerar que estas son diferentes, tanto del centro como entre sí (Aroca \& Soza-Amigo, 2013). En ese sentido, los próximos cambios en la administración del Estado pueden ser auspiciosos en tanto reducen la distancia entre las autoridades y el territorio, como ocurrirá con gobernadores regionales electos por sufragio en lugar de ser designados por el gobierno central, o una eventual redefinición constitucional en el proceso que se iniciará en abril de 2021. En cualquier caso, son proyecciones que aún no se materializan y que, en sus formulaciones iniciales, enfrentarán el centralismo con competencias y recursos limitados (Montecinos, 2020).

En este artículo evaluamos, desde una investigación cualitativa, el proceso de integración en la comuna de Aysén, en la región del mismo nombre y de Cabo de Hornos, en la región de Magallanes, ambas en el sur de Chile. En la siguiente sección discutimos las nociones de compresión espacio-temporal y de injusticia hermenéutica para plantear un marco interpretativo de las brechas entre las zonas aisladas y el desarrollo a escala nacional. Esta brecha se sustenta en una cuestión posicional y decanta en distintas valoraciones de las implicancias de situarse en las 
periferias. A continuación, presentamos los casos de estudio, las comunas de Aysén y Cabo de Hornos, destacando sus dificultades para ser tratados como una simple extensión del territorio nacional.

Los resultados de la investigación muestran una percepción negativa de los esfuerzos de integración. Estos suelen ser vistos como medidas que ahogan las posibilidades de desarrollo de las zonas aisladas en sus propios términos, porque se les imponen metas que no son fácilmente alcanzables. Pero incluso aquellas que son asequibles implican un desvío de trayectorias que se presentan como más deseables a nivel local. Se enfatiza el carácter indeseado de manifestaciones de esta integración, donde un Estado centralizado muestra su mayor rigidez allí donde no logra ejercer su autoridad con toda claridad. Como resultado, la vida cotidiana en las zonas aisladas se vuelve doblemente desafiante: además de las características físicas, se debe lidiar con una estructura estatal que espera demasiado y no permite desviarse de trayectorias predefinidas.

\section{Decidiendo por los otros}

El componente central del aislamiento territorial, en términos de gobernanza, está dado por la exclusión en la toma de decisiones. Aunque la marginación de actores no es un fenómeno limitado a las zonas aisladas, en estas tiene una base de legitimidad generada por las dificultades de la distancia y por la necesidad de extender los beneficios del desarrollo en las periferias. En el caso de la distancia, hay diferentes formas en los que se da por hecho que no es necesario acceder a lugares remotos para levantar información, recoger expectativas o involucrar a sus habitantes (Morgan, 2004). En otras palabras, los resultados de acercarse al territorio no compensan el esfuerzo de llegar a este. En términos prácticos, encontramos en Chile distintas iniciativas en las que las particularidades territoriales son asumidas como comparables entre sí, como ocurre con la medición de la pobreza. No obstante, si las zonas aisladas son dejadas de lado, ¿̇con qué elementos se realiza la comparación? La legitimidad dada por la distancia involucra un riesgo, y es que al asumir que se conoce estas áreas se reproduce una visión reducida y estática de sus dinámicas.

Respecto a la extensión del desarrollo, nos referimos a otro supuesto: estos territorios son carentes de servicios básicos y su habilitación será siempre bienvenida. Por lo tanto, el involucramiento en procesos de consulta o de priorización local de iniciativas de inversión resultaría innecesario, porque toda intervención será aceptada, pero también porque este tipo de procesos ralentizaría la concreción de las propuestas de desarrollo (Amigo, 2017). Esto se relaciona con una visión lineal del desarrollo territorial, que visualiza en la urbanización y en la 
incorporación formal en cadenas de valor las estrategias para mejorar la calidad de vida. Así, las pretensiones locales de mantención de dinámicas productivas locales tradicionales o las dificultades para desplegar redes urbanas y económicas similares a las de las zonas centrales no tienen cabida en las decisiones, y si lo hacen son vistas como desviaciones.

Estos dos supuestos convergen en la noción de integración territorial en tanto proceso de asimilación de distintas áreas dentro de un proyecto de desarrollo común. Esta asimilación implica estandarizar las distintas partes a integrar, pero no implica necesariamente transformarlas para ser idénticas; lo central en dicho debate es que el proyecto integrador define de manera centralizada los elementos de valor de cada territorio y el modo de aprovecharlos, al mismo tiempo que descarta las particularidades locales que no son de interés, o que simplemente son obstáculos para el despliegue de la integración (Núñez et al., 2010; Santos, 1996). La integración territorial, como proyecto centralizado y vertical, no resuelve la exclusión propia del aislamiento. Aun más, puede exacerbarla, pues dicho proceso no finaliza en una incorporación nominal a una unidad territorial mayor, sino que proyecta relaciones de poder con efectos sobre la construcción de identidades que deben ser abrazadas para participar de alguna cadena en la toma de decisiones y también tiene efectos sobre la proyección de estereotipos que eventualmente facilitan la legitimidad en la exclusión de actores locales.

La marginación de grupos de interés alejados de los centros administrativos ha sido abordada en estudios insulares y rurales. En ellos se plantea que los habitantes de territorios diferentes, no homogeneizados y que se mantienen pendientes en su proceso de integración y asimilación están expuestos a un tratamiento condescendiente. Sin embargo, hay un elemento de mayor profundidad en este tratamiento injusto dado a quienes se encuentran en áreas remotas. La noción de injusticia hermenéutica permite problematizar la incapacidad de las sociedades de reconocer las características de sus miembros y proveerles el espacio para expresarlas (Fricker, 2007). Como resultado, se comete un trato injusto sin que se tenga consciencia de ello, pero donde también inciden las propias capacidades de quienes reciben este trato injusto de reconocerlo como tal.

Podemos acceder a las trazas de estas injusticias en los discursos de quienes se ven involucrados en ellas, particularmente de aquellos que encuentran obstáculos en las estructuras sociales para generar esta problematización (Curato et al., 2019). En las estructuras estatales encontramos procesos de integración de territorios diversos mediante la aplicación de mecanismos y procedimientos estandarizados. Sin embargo, estos han sido constantemente criticados por su poca pertinencia a contextos locales marcadamente diferentes de los centros administrativos. Pero ¿qqué pasa cuando los mecanismos poco pertinentes son precisamente aquellos orientados 
a zonas remotas? En esos casos persiste la injusticia hermenéutica en tanto sus efectos no contribuyen necesariamente a mejorar las condiciones de vida de sus habitantes, ni se dirigen a las expectativas que estos manifiestan en términos de desarrollo local.

El centralismo que caracteriza la institucionalidad chilena muestra en el aislamiento una cara marcadamente injusta. Sus antecedentes están en los supuestos que planteamos más arriba, esto es, que las dificultades de acceso y la urgencia de sus problemas exigen pasar por alto procesos deliberativos y de recolección de expectativas locales. En esta exclusión se genera una ampliación de la brecha entre tomadores de decisión a nivel central y los habitantes de estos territorios. No solo se exacerba una incapacidad de conectar con las demandas locales, sino que desde los territorios se propicia el resentimiento hacia las decisiones centrales, particularmente derivadas del Estado (Cramer, 2016). Esto se debe a que el aparato público concentra funciones políticas de las que se espera representatividad, expectativas que son defraudadas cuando de manera vertical se decide por estos territorios. Pero también se debe a que sus resoluciones son ineludibles. Y la experiencia de otros casos muestra que cuando la acción estatal no logra desplegarse con normalidad toma una forma especialmente inflexible, renuente a modificaciones que puedan horadar su estructura (Alston, 2007; Howard et al., 2016; Koss \& Sato, 2016; Mattheis et al., 2019).

Vale la pena aclarar en este punto que esta aproximación no elabora un juicio de valor, ni plantea que haya una intencionalidad detrás de esta injusticia. Que en el centro de la discusión esté la capacidad de comprender fenómenos locales supone que los fenómenos de exclusión y marginación son usualmente inconscientes. Y que esta injusticia radique en el aparato público debe ser contrastado con los esfuerzos de quienes toman decisiones o generan insumos para una institucionalidad compleja, pues deben lidiar con particularidades de muchos tipos al mismo tiempo: reivindicaciones políticas, afirmaciones identitarias, problemas territoriales y económicos diversos. Por ello, una posible explicación para las omisiones que constituyen esta forma de injusticia es que son llevadas a cabo con la mejor de las intenciones, con una convicción legítima de que es necesario acelerar procesos de integración territorial. ¿Cómo se logra una posición más reflexiva sobre el rol del Estado en la integración territorial? ¿Es compatible la integración con la justicia?

La compresión espacio-temporal es un rasgo de la modernidad, fenómeno que aglutina procesos de racionalización de la actividad humana, donde la tecnología fue el motor de cambios que reemplazó no solo la tradición, sino la escala humana (Vesely, 2004), dando paso a procesos de mayor complejidad, como la globalización y la industrialización. En particular, la compresión espacio-temporal alude a la relativización de las distancias, especialmente a partir del acceso a formas de 
transporte que permitieron ampliar las capacidades de desplazamiento. Así, dos lugares geográficamente distantes logran mantenerse en contacto y establecer una suerte de cotidianeidad compartida, uniéndolos en una misma escala.

Sin embargo, esta compresión no es un fenómeno lineal ni se experimenta del mismo modo en todos los territorios unificados bajo dicha escala. Persiste una diferencia entre quienes pueden acceder a los medios que hacen posible el desplazamiento entre lugares y quienes todavía siguen restringidos por las condiciones geográficas (Warf, 2011). Quienes logran superar la escala humana tienen ante sí no un territorio por descubrir, sino uno donde extender sus propias racionalidades y comprenderlos desde los elementos que les son conocidos (Morgan, 2004). El resultado es una doble lectura del territorio, la del habitante y la del visitante (Tuan, 2007), donde la del primero suele ser compleja, llena de información que no ha sido procesada para hacerla suficientemente inteligible para un observador externo. Mientras, el visitante logra establecer comparaciones con las estructuras que le son propias, identificando desde lo anecdótico aquello que es característico del lugar visitado. Por ello, su lectura es más focalizada, a riesgo de representar de manera reducida las dinámicas que allí ocurren.

No es una sorpresa que las interpretaciones de habitantes de zonas centrales y aisladas sea diferente. La compresión espacio-temporal está concentrada en el centro y se vale del rasgo centrífugo de la modernidad, que despliega sus procesos de racionalización en el territorio e incorpora a sus dinámicas los elementos que encuentran sentido en ellas. El hecho de que los propios habitantes encuentren dificultades para precisar lo que les es propio profundiza esa diferencia. Vale la pena destacar que la dificultad está en precisar, no en identificar o en reconocer, pues lo que está en juego es la capacidad de proveer los insumos para que dicha particularidad sea accesible y valorada por el visitante. Por otro lado, también se interpela a la aplicación irreflexiva de la mirada desde el centro, en términos de injusticia hermenéutica, enfatizando en las distorsiones que inciden en que las decisiones tomadas por autoridades estatales convergen con la noción de resentimiento, dada la desconexión entre el sentido de habitar y los efectos de las decisiones tomadas (Cramer, 2016).

\section{Métodos empleados}

Para analizar los procesos de integración territorial en zonas aisladas se empleó un diseño de investigación cualitativa basado en el estudio de casos, correspondientes a dos comunas situadas en el sur austral de Chile. Son las de Aysén, en la región homónima y de Cabo de Hornos, en Magallanes. Ambas son aisladas según el criterio de la Subsecretaría de Desarrollo Regional y Administrativo (2012), 
y son zonas extremas según la ley 20.655. También son cabeceras provinciales, aunque Cabo de Hornos ocupa por sí sola toda una provincia. Presentan numerosas diferencias entre sí, lo que permite aprovechar la fortaleza del estudio de casos para establecer comparaciones y generalizaciones de sus resultados (Yin, 2009). En términos demográficos (ver Cuadro 1), Aysén tiene una población de 23.959 habitantes que, aunque concentrada en la cabecera comunal, se distribuye en varios otros centros poblados, continentales e insulares, y se encuentra en crecimiento, pero que ha perdido impulso en los últimos años. Cabo de Hornos es una comuna enteramente insular. Aunque se compone de decenas de islas, prácticamente la totalidad de su población se concentra en la cabecera comunal, Puerto Williams, experimentando una importante caída en los últimos años.

Cuadro 1. Caracterización demográfica de Aysén y Cabo de Hornos.

\begin{tabular}{|l|r|r|}
\hline & Aysén & Cabo de Hornos \\
\hline Población (2017) & 23.959 & 2.063 \\
\hline Tasa de crecimiento 2002-2017 & 7,2 & $-8,8$ \\
\hline Tasa de crecimiento 1992-2002 & 17,1 & 24,7 \\
\hline Tasa de crecimiento 1982-1992 & 41,0 & 40,1 \\
\hline Densidad (hab/km2) & 0,8 & 0,1 \\
\hline Población urbana (\%) & 84,8 & 90,8 \\
\hline Población indígena (\%) & 34,1 & 21 \\
\hline Índice de masculinidad & 112 & 168,2 \\
\hline
\end{tabular}

Fuente: elaboración propia con base en Instituto Nacional de Estadísticas (s.f.a, 2003, s.f.b, s.f.c).

La principal unidad de análisis es el discurso. La principal fuente más relevante de información la constituyó el discurso recogido mediante entrevistas semiestructuradas. Estas fueron aplicadas a informantes provenientes de agencias estatales, sindicatos, gremios y a habitantes que por su trabajo tuviesen una experiencia de larga data o de fuerte involucramiento en las redes locales. El sentido de estas entrevistas fue acceder a sus propias concepciones sobre la situación de aislamiento y de integración, así como a sus conocimientos sobre la dimensión histórica (Creswell, 2014). En el caso de Aysén se entrevistó a 21 personas, mientras que para Cabo de Hornos se aplicó este instrumento a 19 informantes. Aunque aplicadas principalmente a residentes de estas comunas, también se contactó a actores de carácter regional, en Coyhaique y Punta Arenas, respectivamente, como una manera de abarcar una escala superior en términos de administración territorial, con énfasis en actores del sector público con injerencia en inversiones sectoriales en temas de conectividad, de dinámicas políticas y de desarrollo urbano y productivo. La información en terreno fue recogida entre noviembre de 2017 y 
septiembre de 2018, con dos visitas a cada caso. Esta información fue sometida a análisis temático, con la finalidad de generar categorías interpretativas compatibles entre ambos casos. En particular, se abordó dimensiones de caracterización del aislamiento, del rol del Estado en actuar sobre dichas características, del modo en que dichas acciones son interpretadas por los habitantes de estos territorios y de las manifestaciones prácticas que tomaniniciativas centralizadas en territorios particulares.

La comuna de Aysén fue uno de los puntos de entrada para la ocupación de la región y el lugar donde se radicó la institucionalidad chilena en los inicios de la ocupación de esta zona del país (Martinic, 2005), a contar de 1903. En tiempos en que no había caminos, la comunicación con otros territorios se dio por Argentina o por vía marítima, situación que cambió en las últimas décadas del siglo XX, con la construcción de la Carretera Austral. Todavía en 2020 se trata de una región poco conectada, con pocos vuelos diarios y dependiente del tránsito marítimo y con numerosas localidades en distintas condiciones de aislamiento (Subsecretaría de Desarrollo Regional y Administrativo, 2012). Esto ha dado paso a una marcada identidad regional asociada a los pioneros, primeros habitantes del proyecto nacional, quienes se asentaron para conquistar una naturaleza desafiante, debiendo enfrentar también la falta de servicios y escaso contacto con gente de otros territorios (Brigand et al., 2011). Sin embargo, también ha posibilitado una nueva comprensión de lo aislado como aquello que ha sido preservado (Núñez et al., 2014), con un fuerte énfasis en las cualidades naturales de la región como base para proyectos de conservación ambiental, de turismo de intereses especiales y de calidad de vida.

Aysén representa varias características de aislamiento. Parte de su territorio no está conectado directamente con la cabecera comunal por vía terrestre, como ocurre con el valle Exploradores, para cuyo acceso es necesario atravesar varias comunas y provincias para alcanzar todas sus localidades. Su cabecera, además, está entre Puerto Chacabuco, el principal acceso marítimo de la región y Coyhaique, la capital regional. Mientras, la pequeña localidad de Villa Mañihuales se encuentra junto a la Carretera Austral. Las islas Huichas, por otro lado, contienen a poco más de 1.800 habitantes, ubicados a tres horas de navegación hasta el continente y a un recorrido adicional de entre 30 minutos y una hora y media de servicios y comercio. En esta comuna juega un rol la trayectoria histórica del aislamiento: de ser el eje que orientó la ocupación de la región, actualmente está en su periferia -en el sentido de perder el centro de toma de decisiones-, aunque continúa siendo el principal punto de entrada y salida de carga.

Cabo de Hornos, por su parte, ocupa la zona más austral de la región de Magallanes. Si bien esta área ha sido habitada por el pueblo yagán con asentamientos de larga data, su ocupación formal por parte de Chile es más reciente, con una motivación principalmente orientada al ejercicio de soberanía, a fin de evitar 
avances de Argentina sobre la zona. La fundación de Puerto Williams, su cabecera comunal, en 1953 tuvo como objetivo crear un asentamiento en donde reunir a la población yagán, pero también de establecer una dotación permanente de funcionarios navales y sus familias. Ello explica el alto índice de masculinidad comunal y la fuerte presencia de trabajadores dependientes asociados a las fuerzas armadas y la administración estatal (8,7\%, en contraste con el $1,7 \%$ a nivel regional y 4,4\% a nivel nacional) (Servicio de Impuestos Internos, 2015). Esta comuna está mucho más concentrada que Aysén, pero su población está claramente diferenciada entre quienes ocupan cargos públicos en la municipalidad, la gobernación o el poder judicial, la población dedicada a la pesca de centolla, los habitantes yaganes y quienes trabajan en torno a la Armada y que representan a cerca de la mitad de la población comunal (Berghöfer et al., 2008); (Ilustre Municipalidad de Cabo de Hornos, 2012). Esta población suele permanecer por un período máximo de cinco años, cuando son trasladados a otra base naval. Junto con la reciente creación del principal asentamiento de la comuna, delinearon un territorio habitado mayoritariamente por migrantes, la mayor parte de ellos con tres o cuatro décadas en el lugar.

En ambos territorios se genera una situación periférica intensificada, pues son comunas que están en la periferia de regiones aisladas. La conectividad de ambas depende del tránsito aéreo desde sus capitales regionales, el tránsito marítimo, aunque frecuente, toma varias horas en conectar con sus principales puertos (32 horas para conectar Puerto Chacabuco con Quellón y cerca de 30 horas en el caso de Cabo de Hornos a Punta Arenas) y ambos modos están condicionados por las condiciones atmosféricas. Sin embargo, encontramos diferencias en cuanto al transporte terrestre (prácticamente inexistente en Cabo de Hornos) y en la accesibilidad a sus respectivas capitales regionales, pues Aysén se encuentra a poco más de una hora de viaje por un camino asfaltado, mientras que para viajar desde Puerto Williams a Punta Arenas la ruta aérea comercial implica dedicar tres días para realizar trámites, debido a los horarios de los vuelos.

\section{Bases del aislamiento}

La integración territorial de zonas aisladas tiene manifestaciones prácticas que son experimentadas de manera cotidiana por quienes habitan dichos territorios. Sus consecuencias conectan numerosas escalas y representan desafíos institucionales, como la pretensión de unidad del aparato público, que establece un marco común para todo el territorio nacional. También denotan la diversidad de zonas que requieren atención especial, aunque ello ha sido interpretado a nivel local como una caracterización que los pone en desventaja al momento de participar de la toma de 
decisiones. A nivel local destaca la interpretación de este proceso como uno que deja entrever el desconocimiento desde el nivel central por las necesidades y recursos con que se cuenta para abordar la participación política y el desarrollo productivo. En este análisis nos ocupamos de dichos aspectos, centrándonos en las carencias y rezagos definidos por los propios habitantes de estos territorios, en el modo en que perciben elementos de injusticia en la integración territorial y en efectos prácticos en donde las normas definidas con un criterio unitario tienen consecuencias negativas en la cotidianeidad del aislamiento.

En primer lugar, la noción de carencias y de condiciones diferentes, más desafiantes, impuestas por la geografía son rasgos distintivos que son elaborados por los propios habitantes de estos territorios. Ni la región de Aysén ni la de Magallanes cuentan con conectividad terrestre a través de Chile, lo que tiene impactos sobre el costo del transporte de carga -pues involucra mayores tiempos de traslado a través de barcazas, transporte aéreo o el tránsito a través de Argentina- y sobre la continuidad de la infraestructura de telecomunicaciones. Pero también involucra un sentido de lejanía respecto al resto de Chile, precisamente por la condición de insularidad relativa de ambas regiones. El factor climático es también determinante, debido a la vulnerabilidad de la conectividad ante eventos climáticos como lluvias, vientos, nevazones y bajas temperaturas. Así, la percepción de aislamiento es recordada continuamente a través del hielo en las carreteras, rodados, aluviones y temporales. En conjunto, son factores que condicionan la programación para salir de estas regiones.

La región de Aysén no puede conectarse por territorio chileno con el resto de Chile, esa es una condición básica que nos hace inmediatamente distintos. Si le sumas a eso que la conectividad a través de barco o a través de avión está sujeta siempre a las condiciones climáticas, nos hace estar en una posición que es totalmente distinta, desventajosa (Autoridad regional, Aysén).

Lo anterior describe un panorama relativamente general a nivel regional. $\mathrm{Si}$ nos focalizamos en los casos de estudio, y particularmente en sus localidades marcadamente aisladas, la condicionalidad impuesta por la geografía juega un rol definitivamente cotidiano: no solo afecta las posibilidades de salir de la región, sino que dificulta el acceso a las cabeceras comunales y a los servicios básicos. Esto se relaciona con las carencias materiales y de provisión de servicios en zonas poco pobladas o de asentamientos de difícil acceso y conectividad. En esta evaluación, los informantes destacan el efecto que tienen estas carencias para sostener una trayectoria de desarrollo. No contar con condiciones mínimas de saneamiento es señalado en ambas comunas como la principal dificultad para promover dinamismo económico. Sin embargo, tiene también un impacto en las percepciones de los propios habitantes de estos territorios que, al tomar consciencia del rezago respecto 
a otros centros, sean a nivel nacional o regional, construyen una visión compartida de marginación e insatisfacción.

Como es una zona aislada tenemos algunos problemas, de algunos productos, el hecho de querer estar más integrados al progreso del país, de recibir en alguna medida los beneficios del desarrollo (Autoridad regional, Magallanes).

Vivir en una localidad que no tenga agua potable es terrible, y eso genera pobreza. Si no tienes agua potable, no tienes empresas que inviertan. [...] Si hubiera agua potable, quizás estaría instalada una pesquera, plantas de proceso, y así haces crecer la economía local y dinamizas el transporte, todo (Funcionario municipal, Aysén).

El término "aislamiento" es más amplio que solo geográfico o de conectividad. Se produce un aislamiento incluso un poco psicológico donde muchas personas se sienten postergadas, incluso no partícipes del desarrollo que se está dando a nivel nacional e incluso a nivel regional, donde los esfuerzos se concentran de una forma centralizada. Y ese pensamiento se vuelve más social que personal (Funcionario regional, Aysén).

En las definiciones iniciales planteadas por los entrevistados predomina una visión lineal del desarrollo, que es uno de los supuestos esbozados al discutir sobre las orientaciones de la integración territorial. Esta linealidad es al mismo tiempo verticalidad, en el sentido de que impone hacia escalas subnacionales una manera de comprender el territorio que entiende el desarrollo y la modernización como valores o recursos disponibles en el centro que deben ser extendidos a las periferias (Morgan, 2004). Al profundizar sobre el modo de abordar las carencias y las particularidades geográficas resalta el llamado a un proceso de integración diferenciado, que establezca estándares ajustados a las realidades locales, pero, sobre todo, que permita caminos alternativos de desarrollo. Esto pasa por una noción de particularidad, donde surgen como elementos distintivos un entorno natural relativamente poco alterado - al menos en el caso de Cabo de Hornos, aunque la costa de Aysén comparte dicha visión-y una vida social pausada y ajena a tendencias de grandes ciudades. Aunque la mención al costo de la vida es recurrente, particularmente entre quienes residen en localidades aisladas, suele ser considerado como un compromiso, como un factor para tener en cuenta al proponerse residir en ellas, pero no como un elemento determinante para abandonarlas.

Las bondades que existen todavía en términos de estar al margen de ciertas pandemias, y pandemias no solamente desde el punto de vista infeccioso, sino que también en temas de delincuencia. [...] No solamente es una región extrema o aislada, sino que también es una región de privilegio en el sentido 
de que hay muchas bondades que son mucho más potentes para el alma y para el espíritu (Autoridad regional, Aysén).

El costo de vida es alto y al ser alto es donde... Bueno, siempre lo hemos dicho, que el registro de hogares no sea igual al resto del país. Y no creo que sea solo esta comuna. Debe haber varias en la misma situación porque se mide a todas igual. Perfectamente, con el sueldo mínimo, en la V Región [de Valparaíso] se puede vivir. Acá no. Se sobrevive, pero con ayuda [de subsidios] (Funcionaria municipal, Cabo de Hornos).

Sin embargo, en estos territorios se establece una crítica al paternalismo estatal, en tanto genera una relación de dependencia de subsidios, pero no corrige los problemas estructurales que generan esa necesidad. Allí es donde se conectan esas primeras definiciones lineales del progreso con la compresión espacio-temporal (Morgan, 2004; Warf, 2011): la dependencia del Estado determina trayectorias lineales, pues se orientan a cerrar las brechas entre estos territorios y la zona central del país, desaprovechando las oportunidades de nuevas trayectorias que replanteen, por ejemplo, el modo en que se implementa los subsidios de contratación de mano de obra, la cobertura educacional o los estándares técnicos para el despliegue de actividades económicas a nivel local.

Desde la aplicación del huso horario, que está pensado desde una mirada muy centralista, diría que los incentivos a la inversión [...] requieren una tratativa distinta. Los incentivos a los sistemas de capacitación debiesen también requerir una mirada distinta porque es evidente que, en algunas comunas, en algunas localidades, es muy difícil encontrar la cantidad que se requiere para impartir un curso de capacitación. En una comuna con 500 habitantes es muy difícil pensar que vamos a tener 10, 12, 15 ó 20 gásfiter o electricistas, porque es imposible que la demanda absorba esa cantidad de mano de obra especializada (Autoridad regional, Aysén).

Los entrevistados son críticos de las decisiones de las autoridades del nivel central. Su principal argumento es que, tal como se describe en la última cita, no logran aprehender las particularidades de las zonas aisladas. Sus desafíos son de una escala muy específica, y desde una visión muy generalista difícilmente se accede a la variedad de problemas identificados en nuestros casos de estudio. Así, las percepciones centradas en el desconocimiento de las decisiones tomadas a nivel central son frecuentes y también manifiestan una crítica a lo que describen como un trato condescendiente. 


\section{Abordaje centralizado del aislamiento}

El trabajo de campo de esta investigación no abarcó el proceso de estallido social de octubre de 2019 ni la crisis institucional que lo siguió. Son entrevistas recopiladas a través de dos gobiernos de diferentes bloques partidistas. No obstante, en ellas ya es posible identificar cuestionamientos abiertos a la legitimidad de las autoridades estatales y del esquema centralizado con el que se toma decisiones. No son críticas tan profundas como para justificar el desborde de los procedimientos burocráticos y de las reglas democráticas. Al contrario, encontramos numerosas manifestaciones de seguirlos y aplicarlos aun en contra de la efectividad que podrían tener a nivel local. Esta posición crítica tiene una base de resentimiento, derivado de un trato visto como impertinente, poco adecuado a las necesidades locales $\mathrm{y}$, sobre todo, sin perspectivas de que este mejore (Cramer, 2016).

Las autoridades se sientan en una mesa y hacen una ley para todo el país y resulta que cada región tiene sus cosas distintas. [...] Ellos lo hacen porque tienen la autoridad para hacerlo, pero si estudiaran un poquito no se podría hacer porque cada región tiene su historia distinta (Dirigente Pesquero, Cabo de Hornos).

Son los propios entrevistados quienes explican el desconocimiento de la cotidianeidad de las zonas aisladas. Afirman que el problema parte por el escaso atractivo que estos territorios tienen en términos electorales. Su baja población y la dificultad de sus habitantes para acceder de manera frecuente a los centros de toma de decisiones le restarían interés a candidatos y autoridades. En efecto, recorrer las comunas de Aysén o Cabo de Hornos requiere de varios días, y para una campaña electoral o para una visita desde la zona central significa focalizar recursos en un universo de votantes demasiado reducido. A esto se suma el supuesto de que las necesidades inmediatas son muy básicas, centradas en cobertura de servicios, lo que facilitaría una proyección de una visión lineal de desarrollo (Morgan, 2004).

No van a perder su tiempo de venir a conocer la realidad de Puerto Williams por 900 votos si en esos tres días [de viaje a la ciudad] pueden recorrer Punta Arenas, y allá son 80.000 votos, fácilmente, que están en disputa. Su cargo no se va a decidir en Puerto Williams. Se va a decidir conlosvotos de Punta Arenas y, eventualmente, de [Puerto] Natales. Esas comunas las conocen perfecto, pero no necesariamente conocen la realidad de Puerto Williams (Autoridad regional, Magallanes).

Este supuesto se relaciona con la perspectiva del visitante, en los términos descritos por Tuan (2007). Ella plantea que, al enfrentarnos a un contexto novedoso, distinto a aquel en que nos desenvolvemos normalmente, establecemos 
comparaciones para identificar puntos en común. Esto redunda en una interpretación anecdótica de los lugares desconocidos y acentúa las diferencias, facilitando un abordaje concreto de los problemas, pero al mismo tiempo superficial y posicionado en el lugar desde el que se proviene. Al hablar de aislamiento, planteamos que este ejercicio determina una extensión de racionalidades que tienen sentido en territorios conectados, provistos de servicios (Warf, 2011). Sobre todo, desde posiciones que no han generado el resentimiento hacia las decisiones públicas y que no perciben las dificultades que impone la implementación de decisiones que no resultan pertinentes. En este sentido, la compresión espacio-temporal es representada precisamente por la extensión de un estándar a cada confín integrado al territorio nacional (Morgan, 2004).

Todas las medidas paliativas que se incorpora tienen que ver con la conectividad, o sea, entrego un subsidio para conectar a este territorio con este otro territorio, pero no hay medidas ni miradas largoplacistas que hablen de un desarrollo de esa zona aislada en particular. Mi visión es que son puras medidas paliativas para acercar ese territorio al otro, al desarrollado (Funcionaria regional, Aysén).

Además, el trato condescendiente es mencionado recurrentemente cuando se aborda las interacciones entre autoridades del nivel central con habitantes de zonas aisladas. Este trato es representado como una forma de injusticia testimonial (Fricker, 2007), en el sentido de extender prejuicios sobre quienes viven y se desempeñan en estos territorios. Notemos que son prejuicios que derivan de esa visión anecdótica, propia del visitante, que no logra acceder a la cotidianeidad de habitar lugares enfrentados a difíciles condiciones geográficas. Pero también identificamos la injusticia hermenéutica, que se expresa tanto en dicho desconocimiento como en las capacidades locales de articular narrativas que contengan dicho habitar y que logren, posteriormente, volcarlas en una posición política. Las brechas evidentes entre las autoridades de nivel central y aquellas situadas en regiones extremas tienen una trayectoria (Bustos \& Román, 2019; Cramer, 2016). Han sido construidas a través de décadas de integración y de homologación de las reglas del juego y son especialmente notorias al considerar a quienes residen en localidades que presentan este aislamiento de manera especialmente aguda.

[L]a persona que exponía [venía del nivel central], la primera cosa que dice es "yo pensé que no iba a venir nadie y está lleno de gente, qué bueno que en estos lugares la gente se interese por estos temas". Partió mirándonos a huevo [de manera despectiva]. Ahí uno se da cuenta la visión que tienen desde el norte hacia el sur, cómo nos ven (Funcionaria regional, Aysén) 
Muchas personas te comentaban "esa gente no quiere trabajar, no quiere salir adelante”. Típico de la gente que habla mal, casi que por flojera la gente no quiere hacer las cosas. Y yo llegué y me di cuenta de que no era flojera. Era que en ese entonces no había simplemente Internet. Y todas las postulaciones eran a través de Internet. [...] El propio sistema te lo impedía (Autoridad regional, Magallanes).

A falta de una articulación clara sobre la experiencia del aislamiento identificamos percepciones que denotan incomodidad, resentimiento y frustración (Cramer, 2016). Si bien en algunos casos se habla de abandono para describir la relación con el Estado, los entrevistados reconocen que pese a las falencias hay una inversión importante que se refleja en una cierta flexibilización de los criterios de rentabilidad social para financiar infraestructura de servicios básicos y de conectividad. También reconocen que los subsidios de transporte y salariales contribuyen a paliar el costo de vida en estos territorios. De ahí que una definición más precisa de la relación con el aparato público se acerque a la de marginación o exclusión, porque lo que está en juego es la invisibilización de las realidades de las zonas aisladas. En consecuencia, se establece una frágil vinculación con los procesos de integración territorial, principalmente porque no se percibe que se convoque a sus habitantes a participar activamente del devenir del país. ¿En qué se fundamenta esta sospecha? En que no son considerados de manera activa a nivel local, ni reciben un trato similar al de habitantes de zonas centrales, especialmente al momento de participar de decisiones públicas.

No es que no tomes en cuenta el diagnóstico, [sino que] no estás tomando en cuenta la opinión de la gente, y sigues tomando decisiones en función de los prejuicios que tieneelabogado de la subsecretaría (Funcionaria regional, Aysén).

Estas son las injusticias del desarrollo, del crecimiento, de algunas políticas mal aplicadas. Y muchas veces tampoco existe la voluntad. Contra eso nosotros peleamos, porque está todo centralizado en el gobierno central, en Santiago, y de allá se toma todo (Autoridad regional, Cabo de Hornos).

La integración de las zonas aisladas es percibida como un ejercicio que no contribuye necesariamente al bienestar de estos territorios. No es una percepción que proponga un juicio de valor -por ejemplo, una intención expresa por excluirlas del debate público, o una orientación productiva o de soberanía queinvolucre generar zonas de sacrificio en estos lugares-; más bien, plantea que recurrentemente genera problemas adicionales a los que ya existen por las condiciones de aislamiento. Es la consecuencia de un régimen de gobernanza que impone un estándar para todo el país, pero que no ha evaluado si es apropiado impulsar un desarrollo estandarizado. 


\section{La integración como vulnerabilidad}

Un rasgo característico del aislamiento es la informalidad (Núñez et al., 2017; Tapia, 2015). La necesidad de responder rápidamente a desafíos no previstos por la institucionalidad lleva a la adopción de estrategias que sobrepasan lo normado. Aunque este aspecto puede ser romantizado asociándolo a la capacidad de transformar y acomodar las reglas del juego para sacar lo mejor de un territorio, en la práctica es un factor de vulnerabilidad. La desviación es castigada y en ello se expresa a su vez un rasgo del centralismo y es que en condiciones extremas el Estado no es más flexible. Al contrario, es percibido como más duro, porque a nivel local no se cuenta con los medios para revertir marcos poco pertinentes. Como mucho, se puede apelar a la laxitud del Estado, esto es, que, al no poder desplegarse de manera formal, se omite de actuar. Pero, nuevamente, no corresponde a una acciónqueremedie la falta de pertinencia de un proceso integrador definido de manera estandarizada.

La Contraloría objetó que estábamos financiando particulares para asistir a reuniones y nohabíacómomoverlos. Este año se ha realizadomuy pocas reuniones, y han sido a través de videoconferencias. [Los participantes] se sienten súper ofuscados con esta cuestión, porque noescomo estar en el lugar y las reuniones se hacen en Puerto Montt. Claro, siempre en desmedro de las regiones que son más chicas (Funcionario regional, Aysén).

Hay mucha burocracia en la administración del Estado, hay muchas trabas. Cuando uno quiere hacer algo, impulsar alguna cosa, o se puede, o no se puede, o se tiene que inhabilitar, o está prohibido. Es frustrante, muchas veces, querer hacer cosas por la zona, por la provincia (Autoridad regional, Magallanes).

La vulnerabilidad no se expresa solo en una exclusión que se vuelve recurrente, porque no resulta sencillo involucrar a la ciudadanía de zonas aisladas en la deliberación. Es especialmente aguda cuando expone a sus habitantes a la ilegalidad. De este modo, la incapacidad estructural de reconocer las particularidades territoriales se decanta en un trato injusto. Al excluir el conocimiento basal de las prácticas cotidianas para vivir en zonas aisladas se refuerza las bases del trato injusto de estos territorios (Fricker, 2007), pues la transgresión de normas definidas de manera centralizada permite ratificar los prejuicios sobre las incapacidades o desganos locales por integrarse al resto del país. Además, plantea nuevos puntos focales en los que el visitante, al elaborar su interpretación del lugar ajeno, fijará su atención y establecerá lo que es necesario cambiar, en comparación con el lugar de origen (Warf, 2011; Tuan, 2007). Prácticas aparentemente inocuas, como formas de trueque, representan un riesgo, especialmente para quienes desempeñan actividades económicas de pequeña escala, 
porque los expone a sanciones que tienen sentido a nivel central, pero que resultan poco comprensibles en territorios aislados.

La venta del combustible, por ejemplo. Tienes una ley que rige para todo Chile y uno la entiende, resguardamos la integridad de las personas que hacen este tipo de tareas. Eso genera que al no tener lugares donde se venda combustible, comunas donde no tenemos venta legal de combustible producto de la aplicación de esta ley, hace que el clandestinaje sea la única forma de solucionar ese inconveniente (Autoridad regional, Aysén).

Ir especialmente a pescar y vender no era rentable por el poder comprador que tiene el lugar. [...] Pueblo chico, a veces uno le sacaba más provecho traerle una merluza a un amigo de regalo que venderla, porque de repente vas a necesitar cualquier paleteá [ayuda a modo de retribución] (Dirigente pesquero, Cabo de Hornos).

En efecto, las economías locales se ven fuertemente afectadas por las normas definidas a nivel central. Ellas se ven expuestas a dos desafíos que refuerzan una posición marginal. Por un lado, no logran participar en economías de escala porque los volúmenes de producción o extracción de recursos no es suficiente para proveer cadenas de valor. En estos territorios, en particular, se manifiesta en la producción de carne o la pesca artesanal. Los bajos volúmenes no hacen justificable la inversión en infraestructura sanitaria, como la que se requiere para la certificación de carne y mariscos, lo que dificulta incluso una producción orientada al consumo interno. Por otro lado, la lejanía de centros de mayor tamaño implica una desventaja para llevar los productos ante quienes tienen mayor poder comprador. En conjunto, representan escollos para los que, plantean los entrevistados, el Estado no ha logrado ofrecer respuestas. Además, reflejan el carácter desigual de la compresión espacio-temporal (Warf, 2011), pues impone una racionalidad modernizante que ni es contrastada con las demandas locales ni permite a los habitantes de estos territorios imbuirse de su sentido.

Si bien el método de pesca es artesanal, hay un solo comprador y mucha gente que se dedica a venderle a ese solo comprador. No tenemos venta local. [...] Tenemos una sola persona que legalmente tiene sus permisos y vende sus productos. No hay un punto donde uno pueda comprar (Funcionario municipal, Cabo de Hornos).

Nosotros cuántos años hace que no pescamos [merluza] porque no hay poder comprador. Menos mal que nos dieron la pasada para que le podamos vender a los industriales. Si no, ¿qué hacemos? A esos sistemas estamos también aislados, porque no podemos estar todos los días presionando a la gente, hablando con estos señores (Dirigente pesquero, Aysén). 
Especialmente entre los productores locales persiste la noción de que es necesario apoyar la dinamización de actividades económicas a través de la promoción activa de redes, en lugar de subsidios. Estos han sido vistos como un beneficio que finalmente resulta perjudicial, pues la bonificación para facilitar la rentabilidad del sector productivo no apunta a las dificultades de base para participar de la vida económica del resto del país. En otras palabras, estas ayudas financieras, aunque bienvenidas, no facilitan la integración. Al contrario, pueden ser interpretadas como una acción específica de apoyo a las zonas aisladas que no es retribuida en términos de una vinculación sólida con el desarrollo del resto del país. En ese sentido, mientras las zonas centrales mantienen sus trayectorias y acentúan sus brechas con áreas remotas, estas últimas son depositarias de incentivos desaprovechados.

\section{Conclusiones}

El proceso de integración territorial en las comunas de Aysén y Cabo de Hornos ha sido desafiante para quienes habitan estos territorios. Se trata de dos comunas que representan distintas formas de aislamiento, con dinámicas históricas diversas, pero que comparten una implementación desafiante de iniciativas para incorporarlas al patrón de desarrollo del resto del país. Para comprender dicho proceso nos focalizamos en aspectos basales de un tratamiento de zonas aisladas que es entendido como injusto, pese a que tras él hay una orientación al seguimiento de un estándar similar al de otras comunas y regiones del país, pero en donde además hay iniciativas específicas dirigidas a promover su dinamismo económico y social.

En ambos casos identificamos fenómenos similares, relacionados con la definición de aislamiento, percepciones de exclusión y marginación y la constatación de efectos colaterales de la integración territorial. Entre los primeros, se refuerza una parte del supuesto de las carencias materiales de estos territorios. La falta de servicios, sobre todo sanitarios, representa el principal obstáculo para incorporarse plenamente a dinámicas productivas. Sin embargo, también lo son la lejanía, que dificulta el acceso a los mercados y a los centros decisionales, y los pequeños volúmenes con que se participa a nivel local. Esto contrasta con las iniciativas de subvención a las actividades económicas y son los propios habitantes quienes abordan la necesidad de reorientar esos esfuerzos de una bonificación directa a la materialización de infraestructuras y servicios que no estén limitados al tamaño actual de su población. Para que ello tenga sentido, es preciso abordar el otro supuesto, relacionado con la homologación de los desafíos a nivel local. Allí donde de manera más bien superficial se recoge lo particular de las zonas aisladas, se hace necesario redefinir el rol de la integración territorial. 
Las nociones de exclusión y marginación discuten la visión de abandono de las zonas aisladas. Quienes las habitan reconocen el rol del Estado en apoyar el dinamismo de estas localidades. Sin embargo, lo hace de un modo que deja pocas definiciones a nivel local. Por ello, las decisiones que tienen impacto en estos territorios suelen ser cuestionadas por guardar poca relación con el acontecer cotidiano, debido a que son elaboradas con una mirada estandarizadora que niega las particularidades de estos lugares, a la vez que les impone objetivos y ritmos de desarrollo que desbordan las capacidades locales. Este aspecto se conecta con el tercer elemento revisado a través de la discusión sobre la dureza del Estado al momento de abordar el aislamiento. En efecto, el aparato estatal que se despliega en estos territorios no es inflexible. Al contrario, es muy duro en el sentido de no permitir adaptaciones que faciliten el proceso de integración. Como mucho, se trata de un Estado laxo, que se manifiesta cuando no es capaz de desplegarse en propiedad. Sin embargo, cuando lo hace, vuelve vulnerables a sus habitantes, que cotidianamente deben salir de los canales formales para llevar a cabo las tareas más simples.

Aysén y Cabo de Hornos reflejan que el proceso de integración territorial debe pasar por una mayor flexibilidad. Esto implica reconocer los límites de la institucionalidad para volverlos permeables, permitir adaptaciones y fortalecer un posicionamiento al desarrollo nacional basado en competencias territoriales, en lugar de promover subsidios que, eventualmente, generen una percepción de mayor dependencia, menor autonomía y mayor exposición a un trato marcado por prejuicios y expectativas no resueltas a nivel local.

Agradecimientos

El autor agradece el apoyo del proyecto CONICYT/FONDECYT/3170740 en la elaboración de este artículo.

\section{Bibliografía}

Alston, M. (2007). "It's really not easy to get help": Services to drought-affected families. Australian Social Work, 6o(4), 421-435. https://doi.org/10.1080/03124070701671149

Amigo, C. (2017). "No estamos lejos, allá están lejos". Perspectivas locales sobre aislamiento en Aysén: discurso estatal y aislamiento como territorialidad. In A. Núñez, E. Aliste, Á. Bello, \& M. Osorio (Eds.), Imaginarios geográficos, prácticas y discursos de frontera: Aisén-Patagonia desde el texto de la nación (pp. 167-190). Santiago de Chile: Instituto de Geografía, Pontificia Universidad Católica de Chile. 
Arenas, F., Quense, J., \& Salazar, A. (1999). El aislamiento como desafío para el ordenamiento territorial: el caso de las comunas de Chile. Revista de Geografia Norte Grande, 26, 105-111. http://www.icp.uc.cl/html/revista/PDF/RGNG_N26/art12.pdf

Aroca, P., \& Soza-Amigo, S. (2013). Diferencias productivas estructurales entre el centro y la periferia: Magallanes y Arica versus el promedio nacional. Magallania, 41(2), 101-118.

Berghöfer, U., Rozzi, R., \& Jax, K. (2008). Superando la dicotomía entre conocimiento local y global: diversas perspectivas sobre la naturaleza en la reserva de biosfera cabo de hornos. Environmental Ethics, 3o(SUPPL. 3), 578o. https://doi.org/10.5840/enviroethics200830Supplement58

Brigand, L., Peuziat, I., Arenas, F., Escobar, H., Salazar, A., \& Núñez, A. (2011). Multiplicidad de miradas y valorización del aislamiento geográfico en la Región de Aysén. In A. Núñez, F. Arenas, \& A. Salazar (Eds.), El aislamiento geográfico: ¿problema u oportunidad? Experiencias, interpretaciones y políticas públicas (pp. 121-139). Santiago de Chile: Instituto de Geografía, Pontificia Universidad Católica de Chile.

Bustos, B., \& Román, Á. (2019). A sea uprooted: Islandness and political identity on Chiloé Island, Chile. Island Studies Journal, 14(2), 97-114. https://doi.org/10.24043/isj.91

Cramer, K. J. (2016). The politics of resentment: rural consciousness and the rise of Scott Walker. Chicago: The University of Chicago Press.

Creswell, J. W. (2014). Research design: qualitative, quantitative, and mixed methods approaches. Thousand Oaks: SAGE Publications.

Curato, N., Hammond, M., \& Min, J. B. (2019). Power in deliberative democracy: norms, forums, systems. Cham: Palgrave Macmillan.

Fricker, M. (2007). Epistemic injustice: power \& the ethics of knowing. Oxford: Oxford University Press.

Garay, C. (2004). Estados débiles y espacios vacíos: el caso chileno. Security and Defense Studies Review, 4(2), 91-113.

Howard, A., Katrak, M., Blakemore, T., \& Pallas, P. (2016). Rural, regional and remote social work: practice research from Australia. Oxfordshire: Routledge.

Ilustre Municipalidad de Cabo de Hornos. (2012). Actualización Plan de Desarrollo Comunal (Pladeco) 2012-2017 Ilustre Municipalidad de Cabo de Hornos. s.l.: Ilustre Municipalidad de Cabo de Hornos.

Instituto Nacional de Estadísticas. (s.f.a). Resultados Censo 2017: por país, regiones $y$ comunas. Recuperado el 2 de septiembre de 2020, de http://resultados.censo2017.cl/Home/Download 
Instituto Nacional de Estadísticas (s.f.b). Resultados generales censo de población y vivienda. Chile 1992. Santiago de Chile: Instituto Nacional de Estadísticas.

Instituto Nacional de Estadísticas (s.f.c). Censo de población y vivienda 1982, volumen I. Santiago de Chile: Instituto Nacional de Estadísticas.

Instituto Nacional de Estadísticas. (2003). Censo 2002: resultados. Santiago de Chile: Instituto Nacional de Estadísticas.

Koss, D., \& Sato, H. (2016). A Micro-Geography of State Extractive Power: the Case of Rural China. Studies in Comparative International Development, 51(4), 389-410. https://doi.org/10.1007/s12116-016-9228-4

Martinic, M. (2005). De la Trapananda al Aysén: una mirada reflexiva sobre el acontecer de la Región de Aysén desde la prehistoria hasta nuestros días. Santiago: Pehuén Editores.

Mattheis, F., Raineri, L., \& Russo, A. (2019). Fringe regionalism: when peripheries become regions. Cham: Palgrave Macmillan.

Ministerio de Desarrollo Social. (2018). Situación de pobreza: síntesis de resultados Casen 2017. Recuperado el 25 de noviembre de 2020, de http://observatorio.ministeriodesarrollosocial.gob.cl/casenmultidimensional/casen/docs/Resultados_pobreza_Casen_2017.pdf

Montecinos, E. (2020). Elección de gobernadores regionales en Chile: escenarios de cambio en las relaciones intergubernamentales. Revista de Ciencia Política, 121. (En prensa). http://dx.doi.org/10.4067/So718-090X2020005000101

Morgan, K. (2004). The exaggerated death of geography: learning, proximity and territorial innovation systems. Journal of Economic Geography, 4(1), 3-21. https://doi.org/10.1093/jeg/4.1.3

Núñez, A., Aliste Almuna, E., \& Bello, Á. (2014). El discurso del desarrollo en Patagonia-Aysen: la conservacion y la proteccion de la naturaleza como dispositivos de una renovada colonización. Chile, siglos XX-XXI. Scripta Nova, 18, 46. https://doi.org/10.1344/sn2014.18.15035

Núñez, A., Arenas, F., Brigand, L., Escobar, H., Peuziat, I., \& Salazar, A. (2010). Territorialización del aislamiento geográfico: criterio ambiental para una nueva representación territorial en la Región de Aysén. Revista de Historia y Geografia, 24, 47-66.

Núñez, A., Arenas, F., \& Salazar, A. (2011). El aislamiento geográfico: un asunto de perspectivas. In A. Núñez, F. Arenas, \& A. Salazar (Eds.), El aislamiento geográfico: ¿problema u oportunidad? Experiencias, interpretaciones y políticas públicas (pp. 15-20). Santiago de Chile: Instituto de Geografía, Pontificia Universidad Católica de Chile.

Núñez, A., Baeza, B., \& Benwell, M. C. (2017). Cuando la nación queda lejos: fronteras cotidianas en el paso Lago Verde (Aysén-Chile) - Aldea Las Pampas 
(Chubut-Argentina). Revista de Geografia Norte Grande, 116(66), 97-116. https://doi.org/10.4067/s0718-34022017000100007

Pons, A., \& Rullan, O. (2020). La isleidad como condicionante de la producción del espacio turístico en las Islas Baleares. Boletín de La Asociación de Geógrafos Españoles, 84, 1-33. https://doi.org/10.21138/bage.2804

Saavedra, G. (2016). La pesca artesanal en el sur austral de Chile: controversias territoriales en el espacio marino-costero. Antropologías Del Sur, 5, 65-83.

Santos, M. (1996). De la totalidad al lugar. Barcelona: Oikos-Tau.

Servicio de Impuestos Internos. (2015). Estadísticas de empresas por rubro económico. Recuperado el 25 de noviembre de 2020, de http://www.sii.cl/estadisticas/empresas_rubro.htm

Soza-Amigo, S., \& Aroca, P. (2010). Oportunidades perdidas en Magallanes. Magallania, 38(2), 89-101.

Soza-Amigo, S., \& Correa, L. (2014). Regiones extremas chilenas y su invisibilidad económica. S Si Somos Americanos, 14(2), 187-216. https://doi.org/10.4067/s0719-09482014000200008

Subsecretaría de Desarrollo Regional y Administrativo. (2012). Estudio identificación de localidades en condiciones de aislamiento 2012. Santiago: Subsecretaría de Desarrollo Regional y Administrativo.

Tapia, M. (2015). Frontera, movilidad y circulación reciente de peruanos y bolivianos en el norte de Chile. Estudios Atacameños, 5o, 195-213.

Tuan, Y.-F. (2007). Space and place: the perspective of experience. University of Minnesota Press.

Vesely, D. (2004). Architecture in the age of divided representation: the question of creativity in the shadow of production. Minneapolis: The MIT Press.

Warf, B. (2011). Teaching time-space compression. Journal of Geography in Higher Education, 35(2), 143-161. https://doi.org/10.1080/03098265.2010.523681

Yin, R. K. (2009). Case study research: design and methods. Thousand Oaks: SAGE Inc. 